\title{
Uniform composition film of hydrophilic colloidal gold nanoparticles and hydrophobic carbazole functionalized fluorene trimers for enhanced fluorescence and stability
}

\author{
LIU LinLin ${ }^{1}$, XIE ZengQi ${ }^{1} \&$ MA YuGuang ${ }^{1,2 *}$ \\ ${ }^{1}$ State Key Laboratory of Luminescent Materials and Devices, Institute of Polymer Optoelectronic Materials and Devices, South China University \\ of Technology, Guangzhou 510640, China; \\ ${ }^{2}$ State Key Laboratory of Supramolecular Structure and Materials, Jilin University, Changchun 130012, China
}

Received January 4, 2013; accepted February 21, 2013; published online June 13, 2013

\begin{abstract}
Compositing gold nanoparticles into conjugated molecules have been developed to be one of the most important approaches to increase stability, since degradation of conjugated materials is now one of the biggest bottle-necks to be conquered before industrialization application. Big-size colloidal gold nanoparticles with strong surface plasma resonance are designed to composite with conjugated molecules, in order to realize effective fluorescence enhancement and stabilization. The uniform composition film of hydrophilic colloidal gold nanoparticles (particle diameter of $30 \mathrm{~nm}$ ) and hydrophobic carbazole functionalized fluorene trimers has been obtained by direct mixing of their aqueous and THF solutions, which is determined by AFM. By the comparison of composition based on fluorene trimers with similar structures, we have found that peripheral carbazole group and molecular size of fluorene trimers play an important role in the balance of incompatible solubility, which is regarded as increasing solubility of fluorene trimers in mixed solvent, connecting AuNP and peripheral carbazole groups, and restraining aggregate of gold nanoparticle. This allows facile hydrophilic gold nanoparticle to disperse uniformly in hydrophobic $\pi$-conjugated host. Our investigations show that fluorescence intensity of composition film is enhanced by 4 folds, and heat treatment $\left(200^{\circ} \mathrm{C}\right.$ for $\left.4 \mathrm{~h}\right)$ for the composition film does not induce the degradation of conjugated backbone without the appearance of low-energy emission band, demonstrating the prominent potency of gold nanoparticles in enhanced fluorescence and stability of conjugated molecules and polymers.
\end{abstract}

conjugated polymer, gold nanoparticle, enhanced fluorescence, stability

Citation: Liu L L, Xie Z Q, Ma Y G. Uniform composition film of hydrophilic colloidal gold nanoparticles and hydrophobic carbazole functionalized fluorene trimers for enhanced fluorescence and stability. Chin Sci Bull, 2013, 58: 2741-2746, doi: 10.1007/s11434-013-5869-0

$\pi$-Conjugated molecules and polymers have been extensively studied over the past few decades for the potential application in optoelectronic devices [1]. Until now, one of the biggest bottle-necks for its industrialization is the stability as long-term utilization. Taking one of the typical conjugated polymer polydialkyfluorene (PDAF) as an example, high quantum efficiency of blue fluorescence make PDAF promising candidates for electroluminescent devices, however, it meets stability problems for application, that blue emitting turns to green during operation for appearing of a green band at $\sim 520 \mathrm{~nm}[2,3]$. Enhancement of luminescence

*Corresponding author (email: ygma@scut.edu.cn) stability and quantum efficiency have been observed in PDAF when composites with gold nanoparticles, which is explained as triplet exciton quenching, metal enhanced fluorescence and energy transfer in presence of gold nanoparticles $[4,5]$.

As a rule in nano-science, the size and size distribution of nanoparticles are of key importance in most of their applications. In gold nanoparticle, the intensity and energy level of the electromagnetic field near the particle surface, which called local surface plasma resonance, is determined by shape, size, dielectric environment of nanoparticles [6,7]. In gold nanospheres, it can be characterized by a strong broad absorption band near $520 \mathrm{~nm}$ that is absent in the bulk 
spectra. The increasing of nanoparticle size experimentally enhances the intensity of plasma resonance, shifts the position of plasma resonance [8], and influencing their interaction with surrounding molecules. Qiao et al. [9] has reported that gold nanoparticle with $5 \mathrm{~nm}$ diameter show little enhancement in device performance, but the one with $15 \mathrm{~nm}$ diameter give nearly $20 \%$ improvement, which is from size dependence.

Depending on the compatibility sought, scientists usually choose hydrophobic gold nanoparticles with small radius (1-10 nm) to composite with $\pi$-conjugated molecules. Now for optoelectronic application, the fluorescence enhancement is mainly less than $50 \%$ with partly restrained degradation [4,5]. But to our knowledge, the best experimental enhancement is more than 1000 folds [10] from gold nanobowtie in single molecule fluorescence and it is generally reported to obtain several folds enhancement of monolayer in surface enhanced fluorescence based on gold nanoparticles modified substrate $[11,12]$. So where does this difference come from and how to promote the performance of conjugated molecule/nanoparticle composites? We believe the surface plasma resonance of gold nanoparticle and its interaction with energy level of conjugated molecule play an important role, which strongly depend on particle size. Thus the basic idea in this paper is introducing hydrophilic colloidal gold nanoparticle with bigger size and more effective surface plasma resonance to composite with organic conjugated molecules in order to reach effective fluorescence and stability enhancement.

For the incompatible solubility, hydrophilic nanoparticle is normally utilized after depositing on substrate $[13,14]$, or via surfactant-assistant phase-transfer in material science $[15,16]$. Traditional phase-transfer methods introduce the third intermediate molecules onto gold nanoparticle surface in order to change the solubility, however it lowers the system purity and its role in optoelectronic performance is difficult to evaluate [17]. Some direct phase transfer and compositing works are also reported in order to keep nanoparticle surface active, and build composite structure exactly, e.g. gold nanorod has been transferred to acetonitrile $/ \mathrm{H}_{2} \mathrm{O}$ mixture to build nanorod chain $[18,19]$. Thus we considered to use direct compositing method, which can lower the impurity introducing as little as possible and some specific interaction or bonding between nanoparticle and conjugated matrix could assist nanoparticle distribute uniformly in conjugated matrix.

Many groups have reported that the connection onto the surfaces of gold nanoparticle to form uniform composites by chemisorption between gold and some sulfur or nitrogen containing ligands such as thiol, amine and so on. However, the chemisorption is not quite effective which remains residual function groups in composites and the role of these function groups in optoelectronics is unknown. On another hand, many heterocyclic molecules are widely applied in materials science and some of them have the same element.
Can these heterocyclic function groups hold hydrophilic gold nanoparticle in organic solvents by similar interaction as thiol and amine? Thus we have tried some different molecules with heterocyclic function groups. In this paper we display the results in a series of carbazole functionalized fluorene trimers, one of which show uniform morphology after composited with hydrophilic colloidal gold nanoparticles. We discuss the origin of uniform dispersion and explore the role of colloidal gold nanoparticles in the efficiency and stability of fluorene derivatives.

\section{Experimental}

\subsection{Materials}

The aqueous colloidal gold nanoparticle is synthesized by the classical Frens method [20]. A volume of $95 \mathrm{~mL}$ of a chlorauric acid $\left(\mathrm{HAuCl}_{4}\right)$ solution containing $5 \mathrm{mg}$ of $\mathrm{Au}$ are refluxed and $5 \mathrm{~mL}$ of $1 \%$ sodium citrate solution is added to the boiling solution. The reduction of the gold ions by the citrate ions is complete after $5 \mathrm{~min}$ while the solution is further boiled for $30 \mathrm{~min}$ and is then left to cool to room temperature. This method yields spherical particles with an average diameter of about $30 \mathrm{~nm}$ as determined by dynamic light scattering (Figure 1(a)), and a strong extinction band at
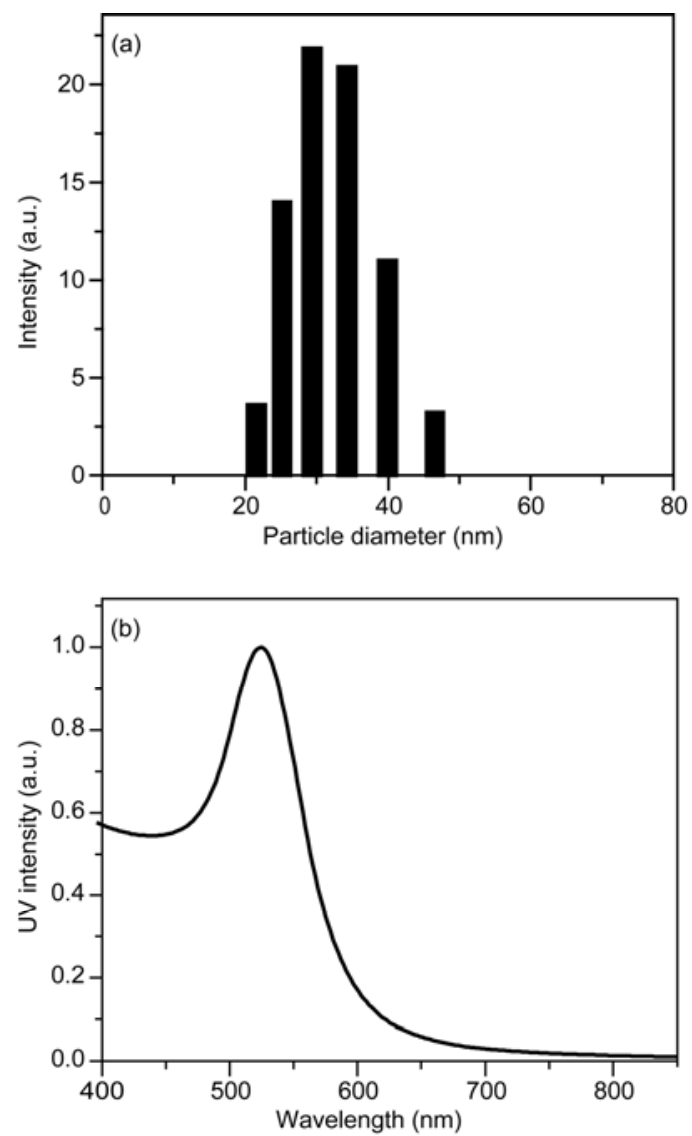

Figure 1 Size distribution histogram (a) and absorption spectrum (b) of AuNP. 
$524 \mathrm{~nm}$ arises from typical surface plasma of gold nanoparticles as shown in Figure 1(b). Three different fluorene trimers with different substituents are chosen to composite with AuNP, as shown in Figure 2. The synthesis and characterization of TFs have been reported in reference [21,22].

\subsection{Instrumentation and methods}

Atom force microscope (AFM) images were recorded under ambient conditions using a Digital Instrument Multimode Nanoscope IIIa operating in the tapping mode. Si cantilever tips (TESP) with a resonance frequency of approximately $300 \mathrm{kHz}$ and a spring constant of about $40 \mathrm{~N} / \mathrm{m}$ were used. The scan rate varied from 0.5 to $1.5 \mathrm{~Hz}$. Fluorescence spectra was obtained on a Shimadzu RF-5301PC spectrophotometer. TFs are firstly dissolved in THF, and AuNP in water is dropped to form composite solution $(\mathrm{TF}=0.1$ $\mathrm{mg} / \mathrm{mL}$, THF $: \mathrm{H}_{2} \mathrm{O}=9: 1$ by volume, $\mathrm{TF}: \mathrm{AuNP}=9: 1$ by weight and $\sim 10^{5}: 1$ by molar). Then the composite solution is spin- coated and drop-cast onto the substrate for AFM and spectroscopy measurement.

\section{Results and discussion}

\subsection{Morphological behavior of composition films based on fluorene trimers with different substituents}

The compatibility sought is one of the key points in composition building, because the phase separation and nanoparticle aggregation are the biggest problems in conjugated molecule/nanoparticle composition. In direct composition, we have tried conjugated molecules with different structures in order to obtain a uniform structure as shown in Figure 2. They all have a spirobifluorene core, and different substituents at 9-position of other two fluorenes. The one with alkyls is given as an inert reference (TF1). Carbazole is connected at the end of alkyl, and two different lengths of alkyl are given as comparison, that TF2 with butylenes and TF3 with hexylenes. We have observed different phenomena when AuNP is composited into fluorene trimers with different subtituents. THF solution of TF1 becomes emulsion immediately after aqueous AuNP dropping, while that of TF2 and TF3 are transparent solutions. Emulsion is normally indicated big aggregates with size larger than the light wavelength, while transparent solutions means solute is well dispersed and solvated as single molecules or small aggregate with size less than the light wavelength.

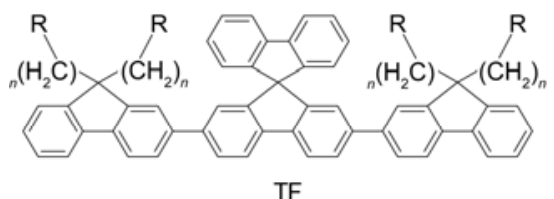

TF1: R=H, $n=6$; TF2: R=Cz, $n=4$; TF3: R=Cz, $n=6$

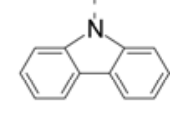

$\mathrm{Cz}$
Figure 2 The chemical structures of fluorene trimers.
We spin-coat the transparent composites solution onto mica substrates and the film morphologies of two composites TF2/AuNP and TF3/AuNP are detected by AFM. Figure 3(a) and (b) shows the AFM height images of (a) TF2/AuNP composite and (b) TF3/AuNP composite spincoated on mica substrates. The smooth background was ultra-thin film spin-coated from $0.1 \mathrm{mg} / \mathrm{mL}$ TF solution; AuNP with $30 \mathrm{~nm}$ size and larger size aggregate is much higher than the background and have been observed in AFM. AFM investigations show big aggregate with size of hundreds nanometer in TF2/AuNP film although its solution
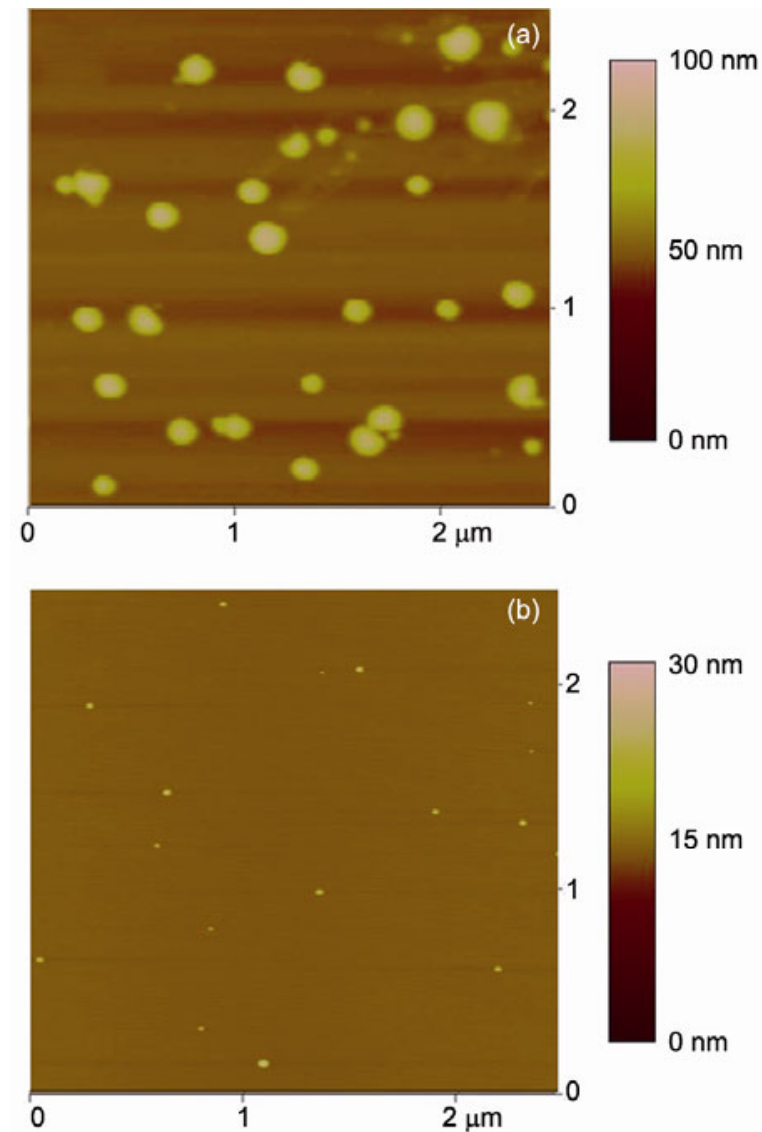

(c)

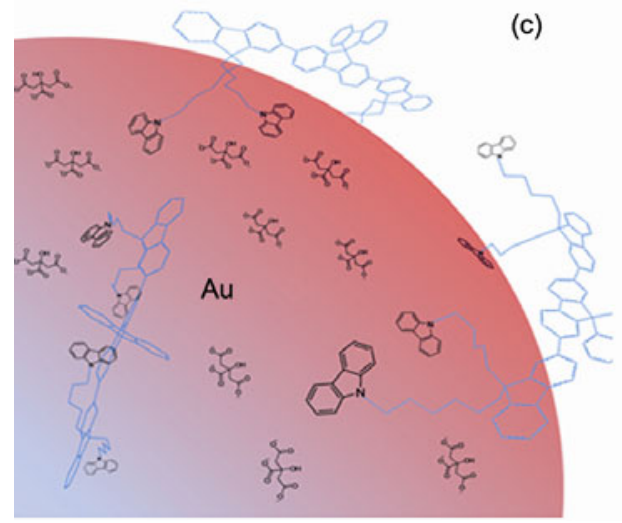

Figure 3 (Color online) AFM height images of (a) TF2/AuNP composite and (b) TF3/AuNP composite spin-coated on mica substrates. (c) Proposed architecture of TF/AuNP nanocomposites. 
is transparent; while TF3/Au composite shows particles in tens nanometer scale dispersed uniformly in conjugated host, which is well corresponding to single AuNP size.

Firstly, the key structural difference between TF1 and TF2 (TF3) is the carbazole at the end of alkyl, thus carbazole would play an important role in the balance of incompatible solubility. The reference tests show that TF1 and AuNP have bad solubility in $\mathrm{THF} / \mathrm{H}_{2} \mathrm{O}$ mixture, while that of TF2 (TF3) is good. Thus the presence of carbazole induces good solubility of fluorene trimers in $\mathrm{THF} / \mathrm{H}_{2} \mathrm{O}$ mixture, and somehow interacts with AuNP to hold it in its bad solvent. It is of importance to determine the nature of interaction between carbazole and Au NP surface. By the comparison of different TFs, we have testified that there is interaction between carbazole and Au NP surface. There would be two possibilities of this interaction to our knowledge: interaction between carbazole and gold metal; interaction between carbazole and citrate on Au NP surface. We have tried to search it by Fourier Transform infrared spectroscopy(FTIR) spectroscopy, and found no signal in mid-IR range, which indicate it is not from interaction between carbazole and citrate. By the limitation of our experimental condition, we cannot measure the interaction between carbazole and gold metal directly, which would be in far-IR range. Thus we suggest the interaction between carbazole and gold metal, which would be similar to amine as a linker connected with Au. Figure 3(c) shows the proposed architecture of TF/AuNP nanocomposites. In order to hold AuNP in THF/ $\mathrm{H}_{2} \mathrm{O}$ mixture, some of citrate on AuNP surface is replaced by TF. Nitrogen in carbazole is proposed to connect with $\mathrm{Au}$, which would be similar as that of amine. For the conjugation effect and space effect from two adjacent phenylenes, the interaction between carbazole and AuNP would be much weaker than that with alkylamine; but as we see, it is strong enough to hold 10\% AuNP in $\mathrm{THF} / \mathrm{H}_{2} \mathrm{O}$ mixture. Especially, carbazole is function group for emission and charge injection/transport [23], but not emission quencher as alkylamine.

Secondly, the only difference between two composites TF2/AuNP and TF3/AuNP is the alkyl structure of fluorene trimer, thus the alkyl length is on duty of the morphological difference, which would be another key point besides carbazole in compositing. Colloidal nanoparticle normally shows large surface energy and tends to aggregate even in its good solvent, unless something big enough on its surface to restrict aggregation. The adsorption of TF would induce a TF monolayer on AuNP surface (Figure 3(c)), and the thickness of this monolayer plays a key role in the aggregate restraining, which would be the reason that longer alkyl length (TF3) shows better morphology without big aggregate.

\subsection{Efficiency and stability in composition film of fluorene derivative and AuNP}

The role of colloidal gold nanoparticles in the efficiency and stability of fluorene derivative are explored by spectroscopy. Theoretically, AuNP tends to enhance absorption and emission efficiency at the same time [24], but in experiment, the absorption is easy to be disturbed by the extinction of gold nanoparticle and difficult to detect. Thus we just compared photoluminescence here, which would be the total affection from both enhanced absorption and emission efficiency. The photoluminescence spectra in solution show typical characteristics of fluorene trimers as shown in Figure 4, no intensity and shape change before and after AuNP doping. It is no surprise to see this similarity for AuNP can only affect the connected TF molecules in solution.

Figure 5 displays the original and normalized (inset) PL spectra of TF2, TF3 and their composites with AuNP in cast film. In normalized spectra, the spectral shapes are superposed well before and after AuNP doping and the main PL peaks for the nanocomposites were located at the same wavelength as that of pure TFs, which indicated that AuNP play little role of the conformation and packing mode of conjugated matrix. It is because the size of $\operatorname{AuNP}(\sim 30 \mathrm{~nm})$ is much larger than that of fluorene trimer $(\sim 2 \mathrm{~nm})$, and matrix diluting affection would be weak. The packing mode

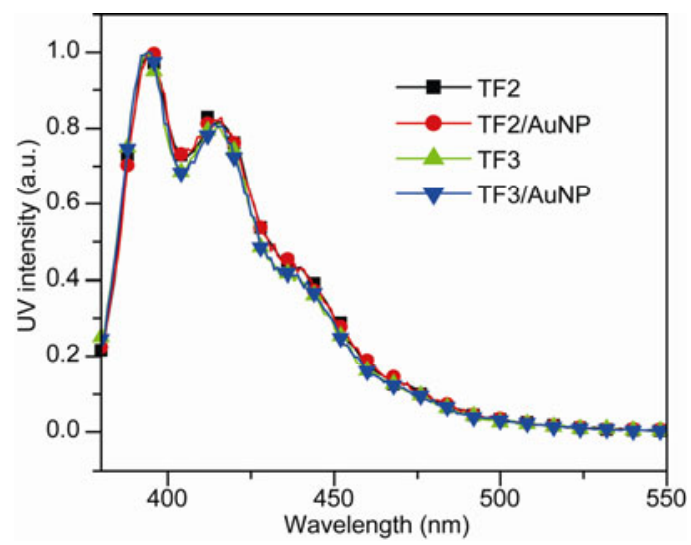

Figure 4 (Color online) PL spectra of TF2, TF2/AuNP, TF3, and $\mathrm{TF} 3 / \mathrm{AuNP}$ in dilute $\mathrm{THF} / \mathrm{H}_{2} \mathrm{O}$ solution.

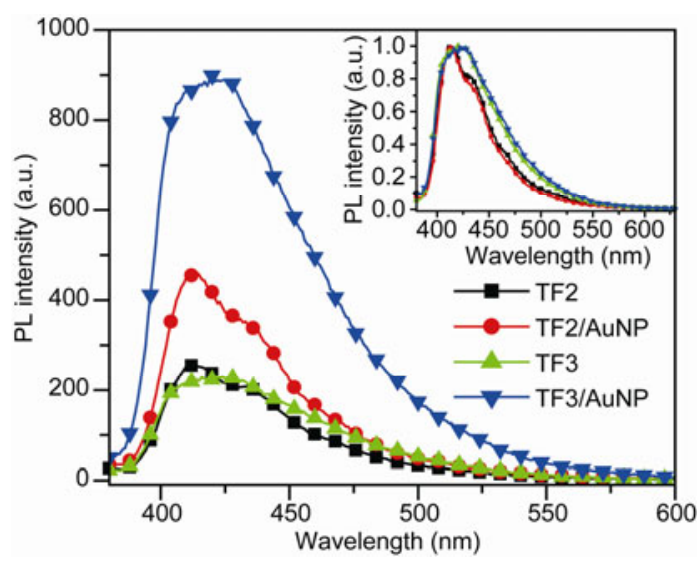

Figure 5 (Color online) Original and normalized (inset) PL spectra of TF2, TF3 and their composites with AuNP in cast films. 
and spectral shape is a little different between TF2 and TF3, which would come from different length of sidechains, although their spectra in solution are the same (Figure 4).

In original spectra (Figure 5), absolute PL intensity is compared before and after AuNP doping. With the same experimental condition, the PL intensity in pristine TF2, TF3 film is similar, while it increases 2-4 times after AuNP doping. It is a remarkable enhancement in thin film of organic nanocomposites, for the best enhancement by organic soluble gold nanoparticle is only reported near 50\%, which is still regarded as partly from matrix diluting and aggregation controlling [5]. This effective enhancement by colloidal gold nanoparticle presumably results from the good size, narrow size distribution and surface properties with effective surface plasma resonance (Figure 1). It has reported that colloidal-gold-nanoparticle based rough substrate show effective surface enhanced fluorescence for single molecules and monolayer [11]. In thin film, the whole integral fluorescence intensity is a statistic value from all the emitting molecules with different distance and dipole orientation, thus it is more significant for material science than that in single molecule or monolayer, and has potential applications of functionalized solid thin film. The different fluorescence enhancement for the cases of TF2 and TF3 indicates the important role of the length of sidechains. The length of the alkyl chains firstly determine the distance between AuNP and the first connecting layer of TFs (Figure $3(\mathrm{c})$ ) that is a key point for surface plasma resonance, and secondly result in different film morphology that is TF2 with shorter alkyl chains tends to form big aggregates with fluorescence quench.

The role of AuNP in stability of TFs is evaluated by thermal annealing experiments. Enhanced efficiency and stability are two typical phenomena in surface plasma effect. In the case of TFs, the thermal annealing evidence would be more powerful, because the degradation induces new emission band at $510 \mathrm{~nm}$ [25]. Figure 6 shows the emission spectra of TFs and the corresponding AuNP composites before and after annealing in air at $200^{\circ} \mathrm{C}$ for $4 \mathrm{~h}$. The thermal degradation of TFs without AuNP is a typical phenomenon of fluorene based degradation. Blue bulk emission decreases and blue shifted, and low-energy emission bands at about $510 \mathrm{~nm}$ are emerging, which is explained as fluorene unit oxidized to emissive fluorenone. When AuNP added, the low energy emission cannot be clearly observed in given degradation period. We defined the intensity ratio $(r)$ in order to compare the degradation degree in different samples:

$$
r^{4 \mathrm{~h}}=I_{\text {green }}^{4 \mathrm{~h}} / I_{\text {blue }}^{4 \mathrm{~h}},
$$

where $r^{4 \mathrm{~h}}$ is the relative intensity of low energy emission at $200^{\circ} \mathrm{C}$ for $4 \mathrm{~h}$ normalized by the corresponding bulk blue emission. As shown in Table 1, the relative intensity of low energy emission $r^{4 \mathrm{~h}}$ are more than 2 in pristine film of TFs, while that with AuNP are less than 0.15, which indicate the

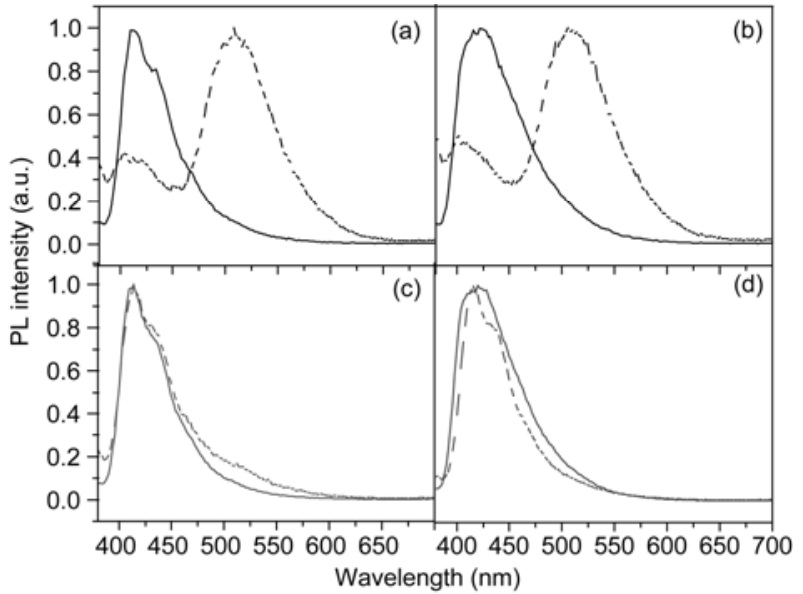

Figure 6 Normalized spectra of (a) TF2, (b) TF3, (c) TF2/AuNP and (d) TF3/AuNP in cast film before (solid state) and after (dash line) annealing in air at $200^{\circ} \mathrm{C}$ for $4 \mathrm{~h}$.

Table 1 The relative intensity of low energy emission

\begin{tabular}{ccccc}
\hline & TF2 & TF3 & TF2/AuNP & TF3/AuNP \\
\hline$r^{4 \mathrm{~h}}$ & 2.35 & 2.04 & 0.15 & 0.11 \\
$R$ & 23.5 & 12 & 2.1 & 0.7 \\
\hline
\end{tabular}

presence of $10 \%$ AuNP increase TF stability more than 13 times. In order to compare the degraded spectrum with the corresponding pristine one, we defined another intensity ratio $(R)$ :

$$
R=\frac{r^{4 \mathrm{~h}}}{r^{0 \mathrm{~h}}}=\frac{I_{\text {green }}^{4 \mathrm{~h}} / I_{\text {blue }}^{4 \mathrm{~h}}}{I_{\text {green }}^{0 \mathrm{~h}} / I_{\text {blue }}^{0 \mathrm{~h}}},
$$

Where $R$ is $r^{4 \mathrm{~h}}$ normalized by a similar parameter in corresponding pristine film. Thus $R$ allow us to estimate the anti-oxidation ability of AuNP, especially when the oxidation process is restrained totally, $R$ would near to $\sim 1$. As shown in Table $1, R$ is only 2 in TF2/AuNP, and 0.7 in TF3/AuNP, although more than 10 in TFs pristine films, which indicate an effective anti-oxidation and well stabilization by AuNP. In TF3/AuNP, $R$ is near to 1 that the intensity of low energy emission are similar before and after degradation (a little less than 1 for the morphology change at high temperature), which means little degradation of TF3 in presence of AuNP in a serious condition of $200^{\circ} \mathrm{C}$ for $4 \mathrm{~h}$.

\section{Conclusions}

In summary, we have uniformly composited hydrophilic gold nanoparticles with big size and strong surface plasma resonance into hydrophobic fluorene trimers. The presence of AuNP shows effective enhancement in the PL intensity and stability, which testify again the basic principle in nano science that the properties strongly depends on the quality of nanoparticle. By the comparison of composition based on 
a series of fluorene trimers with various structures, peripheral carbazole group and molecular size play important role in the balance of incompatible solubility, which is regarded as increasing solubility of fluorene trimers in mixed solvent, weak interaction between AuNP and peripheral carbazole groups and restraining aggregate of gold nanoparticle. More methods to introduce high-quality nanoparticle into conjugated materials uniformly is under searching.

This work was supported by the National Natural Science Foundation of China (20834006, 50873043, 91233113), the National Basic Research Program of China (2009CB623605, 2013CB834705), Guangdong Natural Science Foundation (S2012030006232) and Introduced Innovative R\&D Team of Guangdong (201101C0105067115).

1 Friend R H, Gymer R W, Holmes A B, et al. Electroluminescence in conjugated polymers. Nature, 1999, 397: 121-128

2 Yan M, Rothberg L J, Papadimitrakopoulos F, et al. Defect quenching of conjugated polymer luminescence. Phys Rev Lett, 1994, 73: 744-747

3 Scherf U, List E J W. Semiconducting polyfluorenes-Towards reliable structure-property relationships. Adv Mater, 2002, 14: 477-487

4 Park J H, Lim Y T, Park O O, et al. Polymer/gold nanoparticle nanocomposite light-emitting diodes: Enhancement of electroluminescence stability and quantum efficiency of blue-light-emitting polymers. Chem Mater, 2004, 16: 688-692

5 Hsu S L, Chou C H, Chen C P, et al. Blue electroluminescence enhancement of poly\{2,7-(9,9'-dioctylfluorene)-co-4-diphenylamino4'-bipenylmethylsulfide \} incorporating side-chain-tethered gold nanoparticles. Adv Funct Mater, 2007, 17: 2534-2541

6 Lance Kelly K, Coronado E, Zhao L L, et al. The optical properties of metal nanoparticles: The influence of size, shape, and dielectric environment. J Phys Chem B, 2003, 107: 668-677

7 Underwood S, Mulvaney P. Effect of the solution refractive index on the color of gold colloids. Langmuir, 1994, 10: 3427-3430

8 Jana N R, Gearheart L, Murphy C J. Seeding growth for size control of 5-40 nm diameter gold nanoparticles. Langmuir, 2001, 17: 67826786

9 Qiao L F, Wang D, Zuo L J, et al. Localized surface plasmon resonance enhanced organic solar cell with gold nanospheres. Appl Energ, 2011, 88: 848-852

10 Kinkhabwala A, Yu Z, Fan S, et al. Large single-molecule fluores- cence enhancements produced by a bowtie nanoantenna. Nat Photon, 2009, 3: 654-657

11 Fort E, Grésillon S. Surface enhanced fluorescence. J Phys D: Appl Phys, 2008, 41: 013001

12 Lakowicz J R, Geddes C D, Gryczynski I, et al. Advances in surface-enhanced fluorescence. J Fluorescence, 2004, 14: 425-441

13 Chen S H, Yu S T, Liou Y Y, et al. Increasing the PLED luminescence efficiency by exploiting the surface plasmon resonance effect. J Electrochem Soc, 2011, 158: J53-J57

14 Fujiki A, Uemura T, Zettsu N, et al. Enhanced fluorescence by surface plasmon coupling of Au nanoparticles in an organic electroluminescence diode. Appl Phys Lett, 2010, 96: 043307

15 Subramanya Mayya K, Caruso F. Phase transfer of surface-modified gold nanoparticles by hydrophobization with alkylamines. Langmuir, 2003, 19: 6987-6993

16 Lala N, Lalbegi S P, Adyanthaya S D, et al. Phase transfer of aqueous gold colloidal particles capped with inclusion complexes of cyclodextrin and alkanethiol molecules into chloroform. Langmuir, 2001, 17: 3766-3768

17 Liu L L, Lu P, Xie Z Q, et al. Role of nonemissive quenchers for the green emission in polyfluorene. J Phys Chem B, 2007, 111: 10639_ 10644

18 Liao J, Zhang Y, Yu W, et al. Linear aggregation of gold nanoparticles in ethanol. Colloid Surf A, 2003, 223: 177-183

19 Pramod P, Thomas K G. Plasmon coupling in dimers of Au nanorods. Adv Mater, 2008, 20: 4300-4305

20 Link S, El-Sayed M A. Size and temperature dependence of the plasmon absorption of colloidal gold nanoparticles. J Phys Chem B, 1999, 103: 4212-4217

21 Tang S, Liu M R, Gu C, et al. Synthesis and electrochemical properties of peripheral carbazole functional ter(9,9-spirobifluorene)s. J Org Chem, 2008, 73: 4212-4218

22 Tang S, Liu M R, Lu P, et al. Fluorene trimers with various 9,9'-substituents: The synthesis, characteristics, condensed state structures, and electroluminescence properties. Org Electron, 2008, 9: 241-252

23 Morin J F, Beaupré S, Leclerc M, et al. Blue light-emitting devices from new conjugated poly( $N$-substituted-2,7-carbazole) derivatives. Appl Phys Lett, 2002, 80: 341

24 Vukovic S, Corni S, Mennucci B. Fluorescence enhancement of chromophores close to metal nanoparticles. optimal setup revealed by the polarizable continuum model. J Phys Chem C, 2009, 113: 121133

25 Liu L L, Tang S, Liu M R, et al. Photodegradation of polyfluorene and fluorene oligomers with alkyl and aromatic disubstitutions. J Phys Chem B, 2006, 110: 13734-13740

Open Access This article is distributed under the terms of the Creative Commons Attribution License which permits any use, distribution, and reproduction in any medium, provided the original author(s) and source are credited. 